STRUCTURAL BIOLOGY

ISSN 2059-7983

\title{
The crystal structure of the tetrameric human vasohibin-1-SVBP complex reveals a variable arm region within the structural core
}

\author{
Akihito Ikeda, ${ }^{\text {a }}$ Seia Urata, ${ }^{\text {a }}$ Tadashi Ando, ${ }^{\text {b }}$ Yasuhiro Suzuki, ${ }^{\text {c,d }}$ Yasufumi Sato ${ }^{c, d}$ \\ and Tatsuya Nishino ${ }^{\mathrm{a} *}$
}

Received 31 March 2020

Accepted 18 August 2020

Edited by G. Kurisu, Osaka University, Japan

Keywords: vasohibin; small vasohibin-binding protein; protein complex; X-ray crystal structure; MD simulations; microtubule modification; VASH1-SVBP complex.

PDB reference: VASH1-SVBP complex, 6lpg

Supporting information: this article has supporting information at journals.iucr.org/d

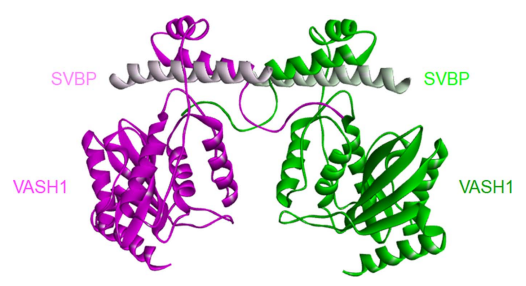
OPEN $\odot$ ACCESS
aDepartment of Biological Science and Technology, Tokyo University of Science, 6-3-1 Niijyuku, Katsushika-ku, Tokyo 125-8585, Japan, 'b Department of Applied Electronics, Faculty of Industrial Science and Technology, Tokyo University of Science, 6-3-1 Niijyuku, Katsushika-ku, Tokyo 125-8585, Japan, ' Department of Vascular Biology, Institute of Development, Aging and Cancer, Tohoku University, 6-6-10 Aramaki Aza Aoba, Aoba-ku, Sendai, Miyagi 980-8575, Japan, and ${ }^{\mathbf{d}}$ New Industry Creation Hatchery Center, Tohoku University, 6-6-10 Aramaki Aza Aoba, Aoba-ku, Sendai, Miyagi 980-8575, Japan. *Correspondence e-mail: tnishino@rs.tus.ac.jp

Vasohibins regulate angiogenesis, tumor growth, metastasis and neuronal differentiation. They form a complex with small vasohibin-binding protein (SVBP) and show tubulin tyrosine carboxypeptidase activity. Recent crystal structure determinations of vasohibin-SVBP complexes have provided a molecular basis for complex formation, substrate binding and catalytic activity. However, the regulatory mechanism and dynamics of the complex remain elusive. Here, the crystal structure of the VASH1-SVBP complex and a molecular-dynamics simulation study are reported. The overall structure of the complex was similar to previously reported structures. Importantly, however, the structure revealed a domain-swapped heterotetramer that was formed between twofold symmetry-related molecules. This heterotetramerization was stabilized by the mutual exchange of ten conserved N-terminal residues from the VASH1 structural core, which was intramolecular in other structures. Interestingly, a comparison of this region with previously reported structures revealed that the patterns of hydrogen bonding and hydrophobic interactions vary. In the molecular-dynamics simulations, differences were found between the heterotetramer and heterodimer, where the fluctuation of the N-terminal region in the heterotetramer was suppressed. Thus, heterotetramer formation and flexibility of the N-terminal region may be important for enzyme activity and regulation.

\section{Introduction}

Vasohibin was first discovered as a factor involved in angiogenesis and is widely conserved among vertebrate species. Two vasohibin paralogs, vasohibin-1 (VASH1) and vasohibin-2 (VASH2), exist in most species, where the former inhibits and the latter promotes neoangiogenesis (reviewed in Sato, 2013). Vasohibins form a complex with small vasohibin-binding protein (SVBP) and are processed and secreted through the noncanonical pathway. Vasohibins and SVBP have recently been found to be involved in the development of neurons and normal basement-membrane formation in the renal corpuscles (Pagnamenta et al., 2019; reviewed in Tanabe et al., 2018). In this pathway, vasohibin participates in the post-translational modification of tubulin, which is known to control neuron differentiation. Both the VASH1-SVBP and VASH2-SVBP complexes function as a tubulin carboxypeptidase to cleave the terminal tyrosine residue, and thus the difference between the two paralogs remains elusive.

Proteases are known to be regulated in multiple stages from their expression to the functional state. For example, serine 
proteases such as trypsin are initially expressed in an inactive zymogen form (trypsinogen) and are subsequently cleaved for activation. Calcium binds tightly to trypsin and stabilizes the protease. Other proteases such as pepsin, gastricsin and renin from the aspartic protease family are also expressed as zymogens and are autoinhibited through a prosegment that precedes the protease domain. The prosegment binds to the protease domain and keeps it inactivated until proteolysis (Dunn, 2002). Vasohibins form a complex with SVBP and complex formation is required for secretion (Suzuki et al., 2010). Sequence analysis of vasohibins identified that they belong to the transglutaminase-like cysteine proteases, with conserved catalytic residues and a putative $\mathrm{Ca}^{2+}$-binding site (Sanchez-Pulido \& Ponting, 2016). Crystal structures of the vasohibin-SVBP complex revealed that the catalytic site is indeed similar to that of transglutaminase (Adamopoulos et al., 2019; Li et al., 2019; Liao et al., 2019; Wang et al., 2019; Zhou et al., 2019; Liu et al., 2019). SVBP binds to the $\mathrm{N}$-terminal structural core region of vasohibin and stabilizes the complex. The structures of complexes with tubulin peptides or inhibitors revealed how vasohibin recognizes substrates (Li et al., 2019; Liao et al., 2019; Wang et al., 2019; Zhou et al., 2019). However, little is known about the dynamics and regulation of the vasohibin complex.

Vasohibins contain less conserved $\mathrm{N}$-terminal and C-terminal regions which are predicted to be disordered and flexible. Previous studies have found that VASH1 is processed at Arg29 and Arg76 (Sonoda et al., 2006). Cleavage at Arg29 can be explained by the disordered structure. In contrast, $\operatorname{Arg} 76$ is part of the SVBP-binding helix, and processing at this residue suggests some dynamic property, but its exact nature remains elusive. We set out to investigate the nature of this region and the structural dynamics of the VASH1-SVBP complex. Surprisingly, we found unexpected heterotetramerization and flexibility within the protease domain which had not been seen in other structures. We performed molecular-dynamics (MD) simulations of the heterotetramer and heterodimer in order to compare their conformational flexibility.

\section{Materials and methods}

\subsection{DNA cloning and protein preparation}

Human VASH1 consists of 364 amino acids, and its molecular weight is $40825 \mathrm{Da}$. Genes for the human VASH1 core (VASH1c) region (amino acids 56-310) and human SVBP (amino acids 2-66) were amplified from pcDNA3.1 and expression vectors (Kadonosono et al., 2017). The PCR fragments were cloned into a modified pMalc2x vector (New England Biolabs). To obtain a soluble stoichiometric VASH1SVBP complex, we created a fusion protein comprising the $\mathrm{N}$-terminal MBP tag, a hexahistidine tag, $5 \times$ TEV proteaserecognition sequence, VASH1 (amino acids 56-310), $2 \times$ TEV protease-recognition sites and SVBP (amino acids 2-66).

For expression of the protein complex, the plasmid (CSBP255) was transformed into One Shot BL21 Star (DE3) pLysS Escherichia coli competent cells (Invitrogen), which were selected on an LB plate with $100 \mu \mathrm{g} \mathrm{ml}^{-1}$ ampicillin. The transformed bacterial colonies were inoculated in LB medium with $100 \mu \mathrm{g} \mathrm{ml}^{-1}$ ampicillin and were cultured at $30^{\circ} \mathrm{C}$ until the $\mathrm{OD}_{600 \mathrm{~nm}}$ reached 0.5. Protein expression was induced by the addition of $0.2 \mathrm{~m} M$ IPTG and incubation for a further $48 \mathrm{~h}$. The bacterial cells were harvested by centrifugation. The bacterial pellet was resuspended in buffer A500 $(10 \mathrm{~m} M$ Tris$\mathrm{HCl}, 500 \mathrm{mM} \mathrm{NaCl}$ ) and was sonicated using a Sonicator XL2020 ultrasonic homogenizer (Misonix) on ice. The lysate was centrifuged at $12000 \mathrm{~g}$ for $15 \mathrm{~min}$. The cleared lysate was applied onto a HisTrap FF column (GE Healthcare) and was eluted with a linear gradient of elution buffer $(10 \mathrm{~m} M$ Tris$\mathrm{HCl}, 500 \mathrm{~m} M \mathrm{NaCl}, 250 \mathrm{~m} M$ imidazole). The eluate was diluted to $100 \mathrm{~m} M \mathrm{NaCl}$ with buffer $\mathrm{A} 0(10 \mathrm{~m} M$ Tris- $\mathrm{HCl} \mathrm{pH}$ 8.0), applied onto an SP Sepharose column (GE Healthcare) and eluted with a salt-concentration gradient using buffer A1000 (10 m $M$ Tris- $\mathrm{HCl} \mathrm{pH} \mathrm{8.0,1} M \mathrm{NaCl})$. The complex was cleaved using a homemade TEV protease. The cleaved product was diluted with buffer $\mathrm{A} 0$ and further purified using SP Sepharose as described above, and the eluate was applied onto a Superdex 200 column (GE Healthcare) pre-equilibrated with buffer A500. The peak fraction was concentrated using an Amicon Ultra-15 centrifugal filter unit $(30 \mathrm{kDa}$ molecular-weight cutoff; Merck). The protein was dialyzed against $10 \mathrm{~m} M$ CHES pH 9.4.

\subsection{Crystallization and structural determination of the VASH1-SVBP complex}

The VASH1-SVBP complex was crystallized by mixing equal amounts of protein solution $\left(17.7 \mathrm{mg} \mathrm{ml}^{-1}\right)$ and solutions from The JCSG Core Suite II crystallization screening kit (Qiagen). The optimal crystal was obtained using the sitting-drop vapor-diffusion method at $20^{\circ} \mathrm{C}$ and a condition consisting of $0.16 \mathrm{M}$ ammonium sulfate, $0.08 \mathrm{M}$ sodium acetate $\mathrm{pH} 4.6,20 \%(w / v)$ PEG 4000, 20\%(v/v) glycerol. Crystals were harvested in the crystallization solution and were flash-cooled under a nitrogen stream. X-ray diffraction data were collected on BL1A at the Photon Factory synchrotron facility (KEK). Diffraction data were processed by XDS (Kabsch, 2010). The structure was determined by molecular replacement using the Phenix package (Liebschner et al., 2019). The coordinates of the human VASH1-SVBP complex (PDB entry 6nvq; Adamopoulos et al., 2019) were used as a search model. The model was refined using iterative modeling and refinement in Phenix and Coot (Emsley et al., 2010). The final model contains VASH1 (chain $A$, amino acids 56-304), SVBP (chain $B$, amino acids 26-53), four sulfate ions and 76 water molecules. Figures were prepared using Coot, BIOVIA Discovery Studio (Dassault Systèmes, Vélizy-Villacoublay, France) and VMD (Humphrey et al., 1996).

\subsection{MD simulation of the VASH1-SVBP complex}

X-ray crystal structures of VASH1-SVBP complexes (PDB entries $6 \mathrm{j} 7 \mathrm{~b}$ and 6ocf and the heterotetramer solved in this study; Liao et al., 2019; Li et al., 2019) were used for MD simulations. To prepare simulation systems of the complexes, 
solvents and small ligands, including an inhibitor, were removed. Using the tleap program in Amber (Case et al., 2018), the complex systems were neutralized with $\mathrm{Cl}^{-}$ions and solvated in a box using the TIP3P water model, where the distance between the water-box wall and the atoms of the proteins was set to $12 \AA$ in the $x, y$ and $z$ directions. Periodic boundary conditions were applied to all simulation systems. The Amber ff14SB force-field parameters were used for the proteins (Maier et al., 2015). The prepared systems were initially minimized first by the steepest-descent method for 1000 steps followed by the conjugate-gradient method for 1000 steps. The temperatures of the systems were then linearly increased from 1 to $310 \mathrm{~K}$ for $100 \mathrm{ps}$ at $1 \mathrm{~atm}$ pressure. After an additional $100 \mathrm{ps}$ MD simulation at $310 \mathrm{~K}$ and 1 atm for equilibration, a $100 \mathrm{~ns} \mathrm{MD}$ simulation was performed to analyze the structural properties of the complexes. A cutoff distance of $12 \AA$ was used for van der Waals and short-range electrostatic interactions, and long-range electrostatic interactions were computed using the particle-mesh Ewald summation method. A 2 fs time step was used for all MD simulations, in which all bonds containing hydrogen were constrained by the SHAKE algorithm (Ryckaert et al., 1977). Structures and energies were sampled every $2 \mathrm{ps}$. The temperature was controlled by the Langevin thermostat (Loncharich et al., 1992) with a $1.0 \mathrm{ps}^{-1}$ collision frequency. The pressure was controlled by the Berendsen barostat

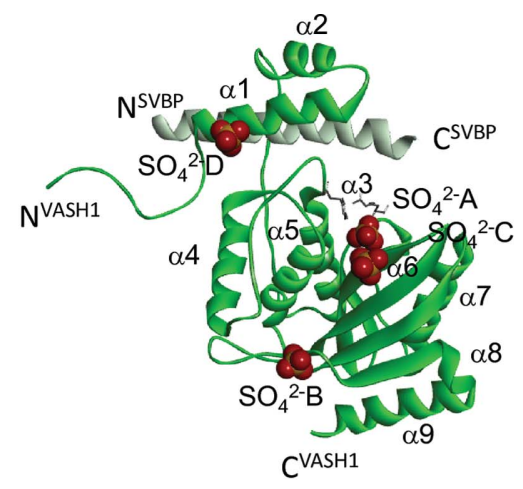

(a)

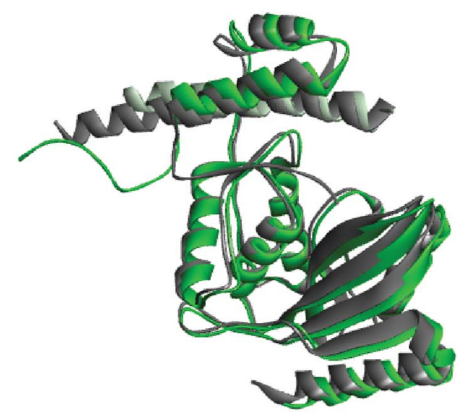

(b)

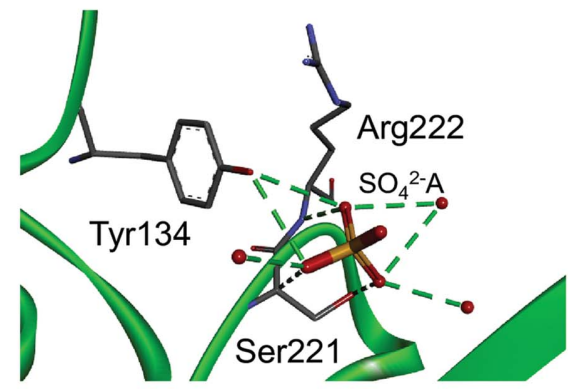

(c)
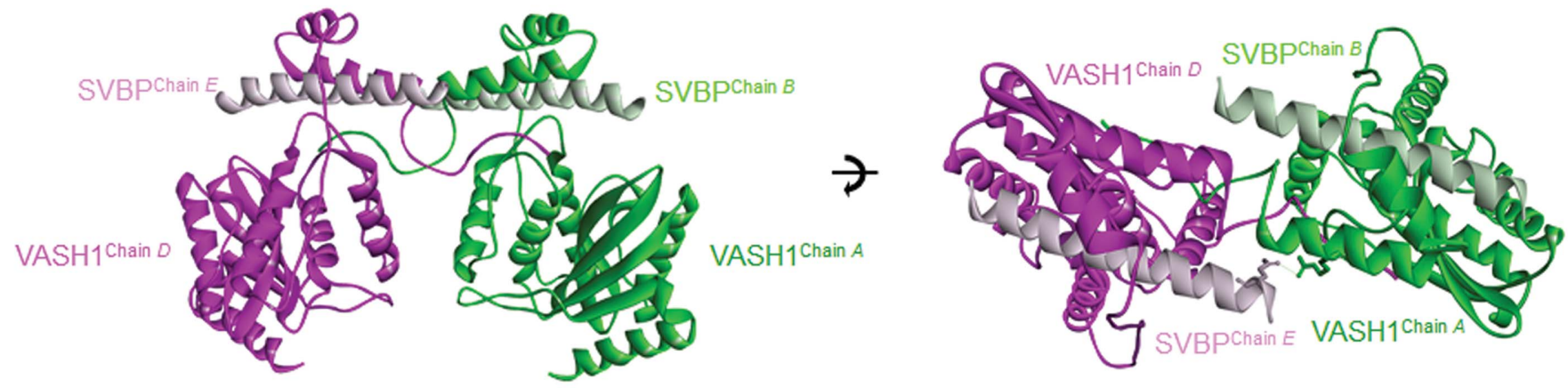

$(d)$

\section{Figure 1}

Crystal structure of the VASH1c-SVBP complex. (a) The VASH1c-SVBP crystal structure in the asymmetric unit. VASH1c is colored green and SVBP is colored light green. Four sulfate ions are shown as spheres and are labeled A-D. Nine $\alpha$-helices of VASH1c are numbered from the N-terminus to the C-terminus. (b) Comparison of VASH1c-SVBP structures. The VASH1c-SVBP structure was superimposed on the reported crystal structure (PDB entry $6 \mathrm{j} 7 \mathrm{~b}$, gray). (c) Sulfate ion $\left(\mathrm{SO}_{4}^{2-} \mathrm{A}\right)$ bound in the vicinity of the catalytic triad. Three residues (Tyr134, Ser221 and Arg222) in contact with the sulfate ion are highlighted as stick models and water $\mathrm{O}$ atoms are shown as red spheres. $(d)$ The VASH1c-SVBP heterotetramer structure in two orthogonal views. A neighboring crystallographic twofold symmetry-related molecule is shown as a ribbon representation. Chains $A$ and $D$ (green and magenta, respectively) correspond to VASH1c. Chains $B$ and $E$ (light green and pink, respectively) correspond to SVBP. Arg76 of chain $A$ (VASH1c) and Glu28 of chain $E$ (SVBP) that form a hydrogen bond to the symmetry-related molecule are shown as stick models. 
(Berendsen et al., 1984) with a pressure-relaxation time of 1.0 ps. For each VASH1-SVBP complex, three independent MD simulations were performed with different random seeds for the Langevin thermostat. For analysis, the first $10 \mathrm{~ns}$ of the trajectories were removed. The root-mean-square fluctuation (r.m.s.f.) of $\mathrm{C}_{\alpha}$ atoms for each complex was calculated after superimposing sampled structures on the average structure. For the heterotetrameric complex, the r.m.s.f. values of chains $A$ (VASH1) and its symmetric chain $D$ (VASH1) were independently calculated to remove the effects of relative motion between them on the local fluctuation. All MD simulations and analyses were performed using the Amber 18 and AmberTools 18 packages (Case et al., 2018).

\subsection{PDB deposition}

The structure of the human VASH1c-SVBP complex has been deposited in the PDB as entry 6lpg.

\section{Results and discussion}

\subsection{Crystal structure of the VASH1c-SVBP complex}

To understand the structural basis of VASH1c-SVBP, we crystallized the purified human VASH1c-SVBP complex. The complex was crystallized in space group $P 6_{1} 22$ and its structure was determined at $2.3 \AA$ resolution by molecular replacement (Fig. 1a, Supplementary Fig. S1, Table 1). The overall conformation of the VASH1c-SVBP dimer was highly similar to the reference structure, with a root-mean-square deviation (r.m.s.d.) of $0.42 \AA$ (Fig. 1b). In the structure, four sulfate ions were bound to the basic surface of VASH1-SVBP. Some of the reported VASH-SVBP structures contained sulfate or phosphate ions bound to the basic surface (Li et al., 2019; Wang et al., 2019). Interestingly, one of the sulfate ions was found in a position close to the catalytic triad and interacted with Tyr134, Ser221 and Arg222 (Fig. 1c). These three amino-acid residues were important in directly recognizing the tubulin peptides and the epoY inhibitor (Liao et al., 2019; Li et al., 2019). Vasohibins are known to bind to the C-terminal tail region of $\alpha$-tubulin, which is rich in glutamate residues. Thus, the bound sulfate ion might mimic the peptide-inhibitor interaction.

While the overall structural features were consistent with the reported structures, we noticed that the $\mathrm{N}$-terminal region (amino acids 56-67) did not align well and protrudes from the VASH1c-SVBP core (Fig. 1b). A comparison of our structure with previously reported structures indicated that none of the reported structures were in the same orientation. Surprisingly, the N-terminal region was inserted into the neighboring crystallographic symmetry-related VASH1c-SVBP molecule to form a heterotetramer (Fig. 1d). The heterotetramer was formed by the crystallographic twofold axis; thus, the $\mathrm{N}$-terminal region of the other molecule was inserted into the symmetry-related molecule.

The superposition of VASH1 structures revealed variation in the conformations of the $\mathrm{N}$-terminal region. There were even some structures in which this region was absent. Among the structures that contain this $\mathrm{N}$-terminal region, we picked
Table 1

Data-collection and refinement statistics for the VASH1c-SVBP complex.

Values in parentheses are for the highest resolution shell.

\begin{tabular}{|c|c|}
\hline Wavelength $(\AA)$ & 1.1 \\
\hline Resolution range $(\AA)$ & $40.76-2.30(2.382-2.300)$ \\
\hline Space group & $P 6_{1} 22$ \\
\hline$a, b, c(\AA)$ & $71.854,71.854,215.811$ \\
\hline$\alpha, \beta, \gamma\left({ }^{\circ}\right)$ & $90,90,120$ \\
\hline Total reflections & 298269 (30582) \\
\hline Unique reflections & $15487(1492)$ \\
\hline Multiplicity & $19.3(20.5)$ \\
\hline Completeness (\%) & $99.88(100.00)$ \\
\hline Mean $I / \sigma(I)$ & $24.93(2.48)$ \\
\hline Wilson $B$ factor $\left(\AA^{2}\right)$ & 48.41 \\
\hline$R_{\text {merge }}$ & $0.09476(1.336)$ \\
\hline$R_{\text {meas }}$ & $0.09735(1.370)$ \\
\hline$R_{\text {p.i.m. }}$ & $0.02208(0.3012)$ \\
\hline $\mathrm{CC}_{1 / 2}$ & $1(0.807)$ \\
\hline $\mathrm{CC}^{*}$ & $1(0.945)$ \\
\hline Reflections used in refinement & $15473(1492)$ \\
\hline Reflections used for $R_{\text {free }}$ & 1548 (149) \\
\hline$R_{\text {work }}$ & $0.1896(0.2300)$ \\
\hline$R_{\text {free }}$ & $0.2362(0.2910)$ \\
\hline $\mathrm{CC}_{\text {work }}$ & $0.957(0.874)$ \\
\hline $\mathrm{CC}_{\text {free }}$ & $0.895(0.749)$ \\
\hline \multicolumn{2}{|l|}{ No. of non-H atoms } \\
\hline Total & 2358 \\
\hline Macromolecules & 2262 \\
\hline Ligands & 20 \\
\hline Solvent & 76 \\
\hline No. of protein residues & 277 \\
\hline R.m.s.d., bonds (£) & 0.007 \\
\hline R.m.s.d., angles $\left({ }^{\circ}\right)$ & 0.86 \\
\hline Ramachandran favored (\%) & 95.97 \\
\hline Ramachandran allowed (\%) & 4.03 \\
\hline Ramachandran outliers (\%) & 0.00 \\
\hline Rotamer outliers (\%) & 4.12 \\
\hline Clashscore & 6.05 \\
\hline \multicolumn{2}{|l|}{ Average $B$ factor $\left(\AA^{2}\right)$} \\
\hline Overall & 60.22 \\
\hline Macromolecules & 60.00 \\
\hline Ligands & 92.05 \\
\hline Solvent & 58.29 \\
\hline No. of TLS groups & 10 \\
\hline
\end{tabular}

two structures (PDB entries 6j7b and 6ocf) that best represent the conformational variations. Superposition of the three structures revealed that the position of Gly65 differed greatly and the side chain of Arg64 faced in opposite directions (Fig. 2a). The N-terminal region preceding cis-Pro68 shows large conformational variations, whereas the region that follows Pro68 similarly forms an $\alpha$-helix and superimposes well. When we measured the angles made by the $\mathrm{C}_{\alpha}$ atoms of Glu71, Pro68 and Arg64, the heterotetramer was most extended, with an angle of $112^{\circ}$. The angles made by the heterodimers are more acute $\left(95^{\circ}\right.$ in PDB entry 6ocf and $74^{\circ}$ in PDB entry 6j7b), and PDB entry 6j7b shows a sharp turn. The comparison of torsion angles indicates a large difference in Gly65 and Gly66, indicating peptide flipping (Table 2). As a result of this structural variation, the region from Gly56 to Arg64 was inserted into the cleft in the VASH1-SVBP core domain (Fig. 2b).

To carefully analyze the difference, we used the PISA server (https://www.ebi.ac.uk/pdbe/pisa/) and BIOVIA Discovery Studio. The interface area was similar in the heterotetramer 
and the heterodimers, but the heterotetramer had more interactions compared with the heterodimers, which might result in a lower solvation free energy (Fig. 3 and Table 3). In the heterotetramer, Val62-Gly66 formed hydrogen bonds to the same region of the symmetry-related molecule. There were also interactions of the inserted N-terminal region with Val69, Met77, Phe142 and Phe141 of the VASH1 core regions (Fig. 3a). Other interactions were similarly observed in the heterotetramer and the heterodimer. Phe60, Phe61 and Arg64 participate in interactions (Figs. $3 a, 3 b$ and $3 c$ ). Notably, PDB entry 6ocf was stabilized by minimal interaction with the VASH1 core regions (Fig. 3c). Thus, our tetrameric crystal structure revealed an unexpected variability of the $\mathrm{N}$-terminal region and its stabilization through extensive interactions.

\subsection{MD simulations of VASH1c-SVBP complexes}

Having found the difference in the $\mathrm{N}$-terminal structure, we performed a $100 \mathrm{~ns}$ MD simulation of the heterotetramer and the heterodimer (PDB entries 6j7b and 6ocf) complexes in triplicate to characterize the conformational flexibility and stability of the N-terminus. The $\mathrm{C}_{\alpha}$ r.m.s.d.s of VASH1 from the initial structures of the three complexes were less than

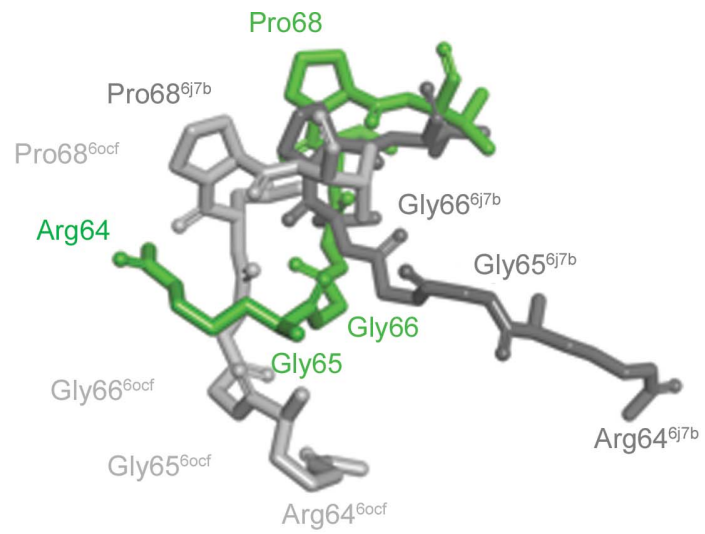

(a)

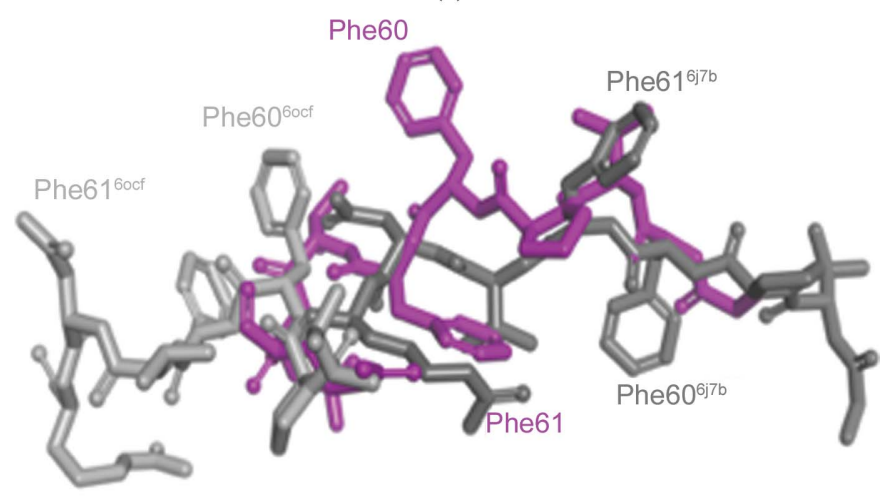

(b)

Figure 2

Conformations of the N-terminal region of VASH1c in three different VASH1c-SVBP complex structures. (a) Close-up view of the VASH1 N-terminal region (Arg64-Pro68) shown as a colored stick model: heterotetramer (chain $A$ ), green; PDB entry $6 \mathrm{j} 7 \mathrm{~b}$, gray; PDB entry 6ocf, light gray. (b) Close-up view of the VASH1 N-terminal region (Gly57Arg64) shown as a colored stick model: heterotetramer (chain $D$ ), magenta; PDB entry 6j7b, gray; PDB entry 6ocf, light gray
Table 2

Comparison of the torsion angles of the three VASH1-SVBP complex structures.

\begin{tabular}{|c|c|c|c|c|c|c|c|c|}
\hline \multicolumn{3}{|c|}{ Residue } & \multicolumn{2}{|l|}{ Tetramer } & \multicolumn{2}{|c|}{ PDB entry $6 j 7 b$} & \multicolumn{2}{|c|}{ PDB entry 6ocf } \\
\hline No. & Name & Type & $\varphi\left({ }^{\circ}\right)$ & $\psi\left({ }^{\circ}\right)$ & $\varphi\left(^{\circ}\right)$ & $\psi\left({ }^{\circ}\right)$ & $\varphi\left(^{\circ}\right)$ & $\psi\left({ }^{\circ}\right)$ \\
\hline & Pro & & & & & & & \\
\hline & & & & & & & & \\
\hline & & & & & & & & \\
\hline & Val & & & & & & & \\
\hline & Asn & & & & & & & \\
\hline & Arg & & & & & & & \\
\hline & & & & & & & & \\
\hline & & & & & & -1 & & \\
\hline & $\mathrm{Le}$ & & & & $3-11$ & & & \\
\hline & Pro & & & & & & & 53. \\
\hline & Val & & & & & & & $+\theta_{0}$ \\
\hline & Asp & & & & & & & \\
\hline & Glu & & -72.44 & -26.88 & -55.91 & -43.05 & -63.08 & \\
\hline
\end{tabular}

Table 3

Interface properties of the N-terminal region of VASH1c with its core region analyzed by the PISA server.

\begin{tabular}{llll}
\hline Structure & N-terminal region & Interface area $\left(\AA^{2}\right)$ & $\Delta G \dagger\left(\mathrm{kcal} \mathrm{mol}^{-1}\right)$ \\
\hline Tetramer & $56-67$ & 551.5 & -7.5 \\
PDB entry 6j7b & $57-67$ & 659.9 & -5.5 \\
PDB entry 6ocf & $58-67$ & 540.0 & -7.0 \\
\hline
\end{tabular}

$\dagger$ Solvation free-energy gain upon formation of the interface.

$3.5 \AA$ throughout the simulation time (Supplementary Fig. S2), indicating that the overall structures of VASH1 are stable for all complexes. VASH1 has five consecutive hydrophobic residues in the N-terminal region, VPFFV at residues 58-62 $\left(\mathrm{VPFFV}_{58-62}\right)$, which contribute to forming the hydrophobic core of VASH1. We calculated the solvent-accessible surface areas (SASA) of $\mathrm{VPFFV}_{58-62}$ in chains $A$ and $D$ of the heterotetramer in PDB entries $6 \mathrm{j} 7 \mathrm{~b}$ and $60 \mathrm{cf}$ as a function of simulation time (Figs. $4 a, 4 b$ and $4 c$ ). Interestingly, the SASA values fluctuate around $350 \AA^{2}$ during $100 \mathrm{~ns}$ for PDB entries $6 \mathrm{j} 7 \mathrm{~b}$ and 6ocf. On the other hand, the SASA value of the heterotetramer remained around $150 \AA^{2}$ for $100 \mathrm{~ns}$ except for one chain in the second simulation. In this exception, the $\mathrm{N}$-terminus of the VASH1 chain detached from the hydrophobic core of VASH1 at an early stage of the simulation and largely fluctuated throughout the simulation (Supplementary Fig. S3 and Movie S1). When we calculated the r.m.s.f. of $\mathrm{C}_{\alpha}$ atoms in the N-terminus of VASH1 in the complexes, the values for the heterotetramer were slightly lower than those for the heterodimers except for the chain in the second simulation, and there were several peaks for the heterodimer which were absent for the heterotetramer. When we analyzed the differences in interaction, the peak at Arg64-Gly65 in the loop region may have been suppressed by the hydrogen bonds that were formed between the backbones of Val62 and Gly66 in the same region of the symmetry-related molecule in the heterotetramer (Fig. 3a). Two other peaks at Glu71 and Arg76 may have been suppressed by hydrogen bonds between Arg76 and Glu28 of the neighboring SVBP (Fig. 1d). Additionally, in the heterotetramer we observed that the orientations of the two dimers changes during the simulation (Supplementary 
Movie S2). As the two dimers are connected mainly through the N-terminal region, there may be a pivotal rotational movement. The results of the MD simulations demonstrate that there is a difference in the flexibility of the $\mathrm{N}$-terminal region between the heterodimer and the heterotetramer.

What are the roles of the $\mathrm{N}$-terminal region (amino acids 56-69) and the significance of the conformational variability? One possibility is that it is necessary for SVBP binding, tubulin binding and cleavage, secretion and activation/inactivation. The previously observed VASH1 processing at Arg76 may be caused by the plasticity of this region (Sonoda et al., 2006). There are several VASH1-SVBP crystal structures without this region; thus, VASH1 can bind SVBP independently and is not involved in complex formation (Liao et al., 2019). Moreover, the VASH1 truncation construct (amino acids 77-365) can inhibit neovascular formation, which indicates that the complex is functional and excludes a role in secretion (Sonoda et al., 2006). It may be that the mobility of the N-terminal region and tetramer formation may negatively control the protease activity of VASH1. Such autoinhibition/processing has been observed for the renin prosegment, which binds and forms a $\beta$-sheet with the protease domain to block the active site (Morales et al., 2012). Interestingly, in the VASH1-SVBPepoY structure Phe60 of the N-terminal region was close to the epoY inhibitor (Liao et al., 2019), but in other structures, the same Phe60 side-chain rotamer is oriented differently ( $\mathrm{Li}$ et al., 2019). In the VASH2-SVBP-epoY crystal structure, the corresponding Phe49 was also close to epoY (Wang et al., 2019). In contrast, truncated VASH1 lacking this region was used in the tubulin complex (Liao et al., 2019), and in the VASH2-SVBP-tubulin complex the side chain of Phe 49 was disordered and was not modeled. Moreover, the flexibility of the $\mathrm{N}$-terminal region was partly suppressed by the formation of the heterotetramer, where the neighboring SVBP contacted the region. This conformation might prevent processing at Arg76. As we have observed a pivotal movement of the

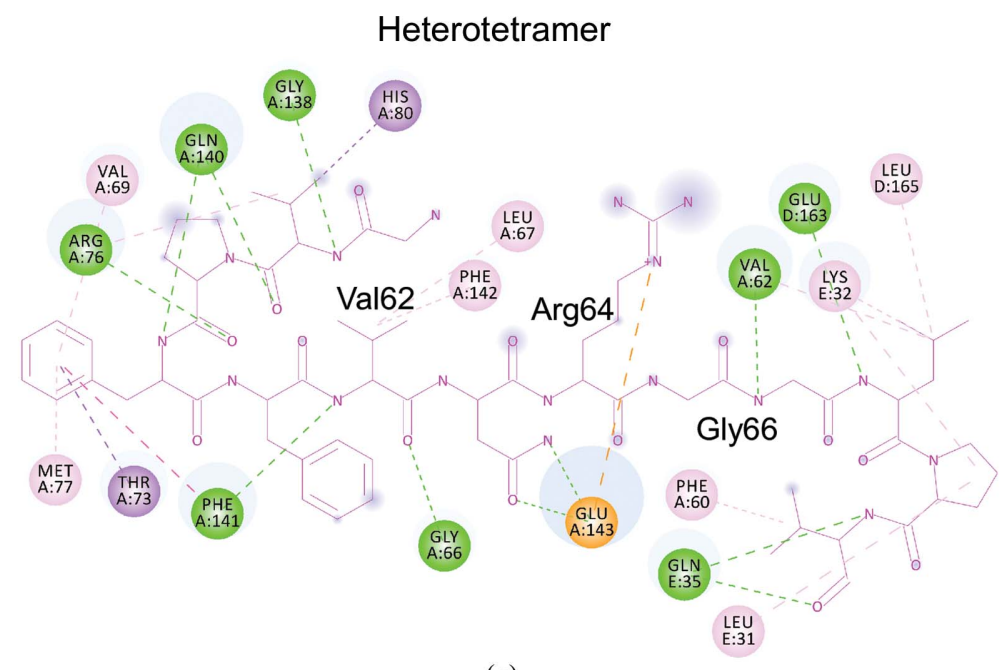

(a)

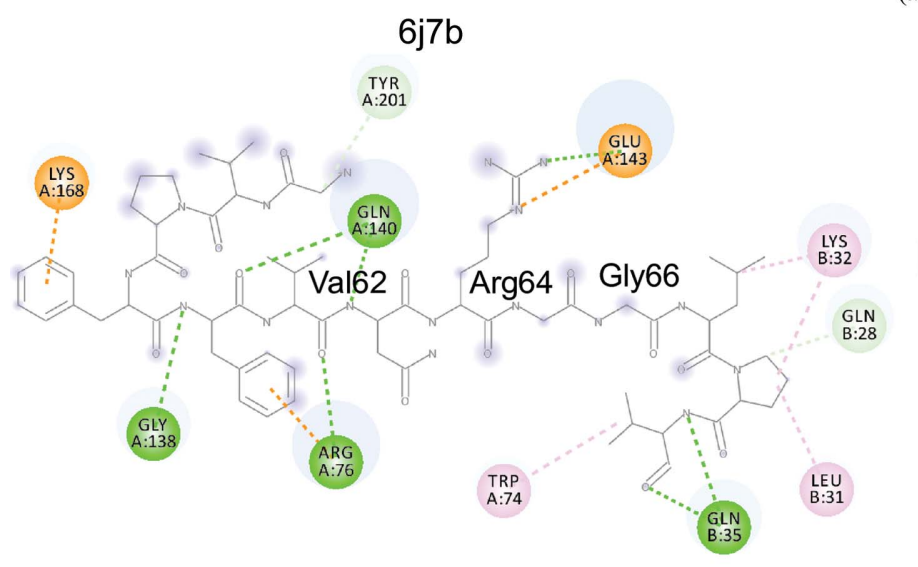

(b)

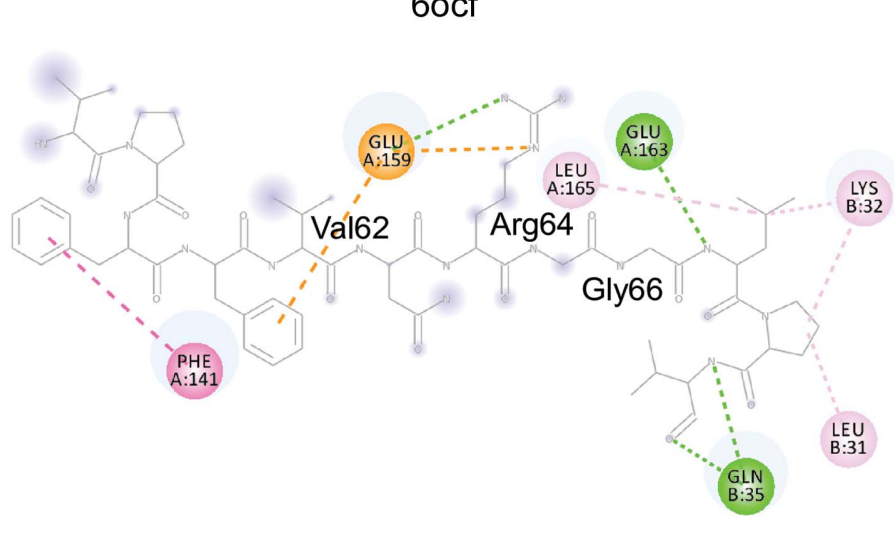

(c)

Figure 3

An interaction map of the VASH1c N-terminal region. (a) Heterotetramer. VASH1 chain $D$ is shown as a structural formula colored magenta. Chains $A$ and $D$ are VASH1 and chain $E$ is SVBP. (b) The PDB entry 6j7b heterodimer. VASH1 chain $A$ is displayed as a structural formula colored dark gray. Chain $B$ is SVBP. (c) The PDB entry 6ocf heterodimer. VASH1 chain $A$ is displayed as a structural formula colored light gray. Chain $B$ is SVBP. Intramolecular and intermolecular interactions of the $\mathrm{N}$-terminal region are analyzed and shown in the 2D diagram. Interacting amino acids are shown in a circle with chain ID and residue numbers. The circles are colored according to the following scheme. Attractive charge interaction, orange; hydrogen bond, green; $\pi-\sigma$ interaction, purple; $\pi-\pi \mathrm{T}$-shaped interaction, magenta; alkyl- $\pi$-alkyl interaction, pink. The size of the blue shadow of an atom or amino-acid residue corresponds to the degree of solvent accessibility. 


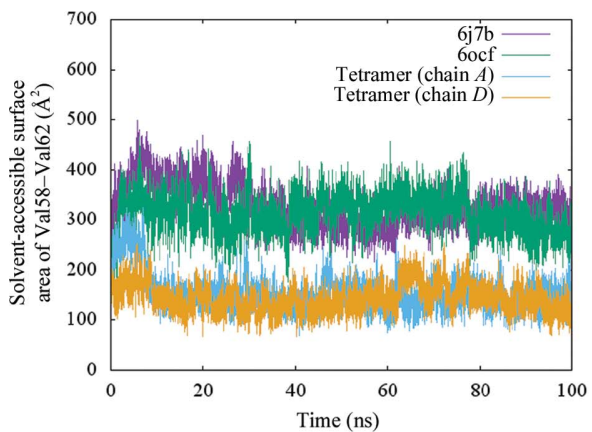

$(a)$

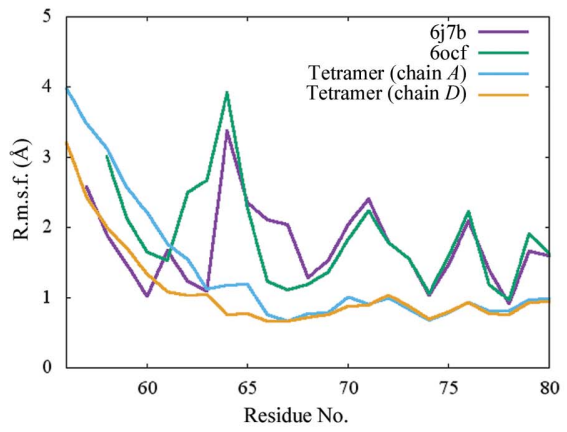

$(d)$

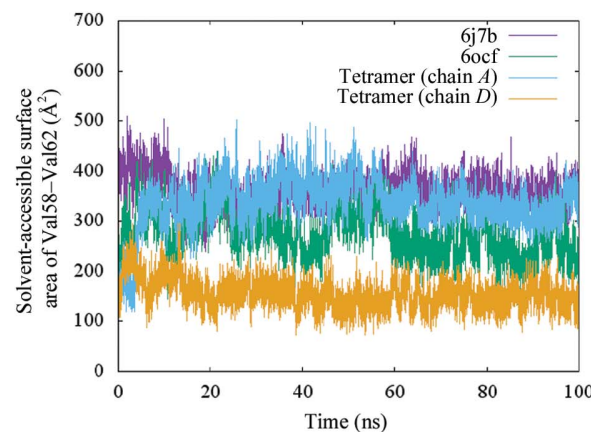

(b)

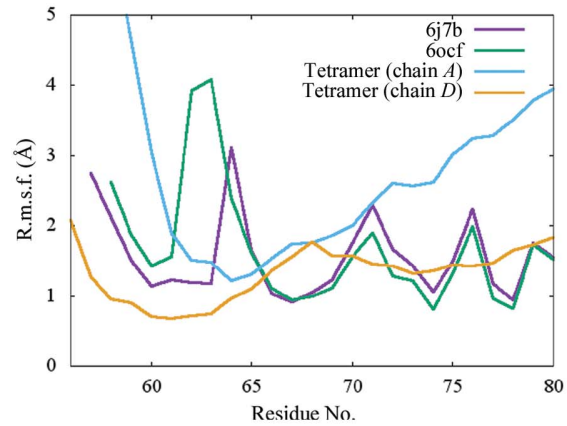

(e)

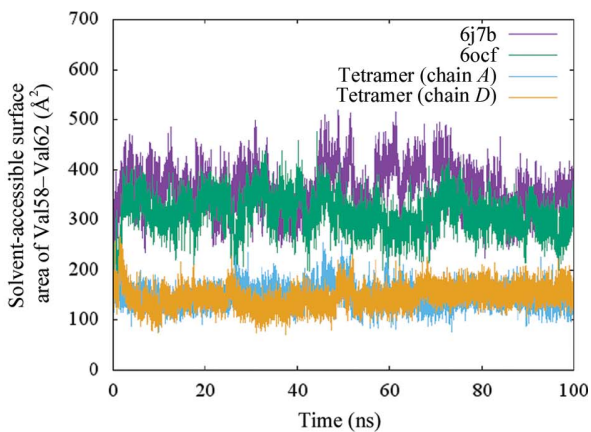

(c)

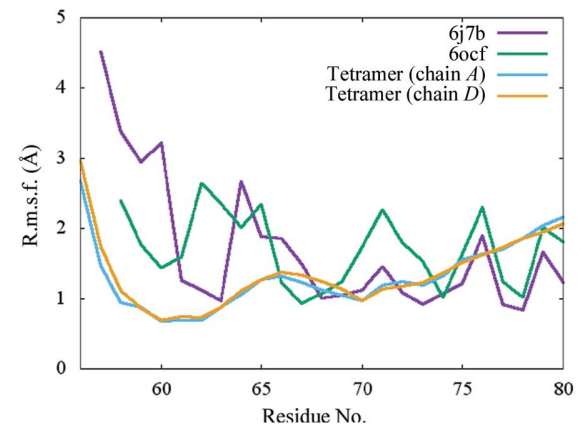

$(f)$

Figure 4

Three independent $100 \mathrm{~ns}$ MD simulations of three different VASH1c-SVBP complex structures. $(a, b, c)$ Time evolution of solvent-accessible surface areas of the hydrophobic moiety $\operatorname{VPFFV}_{58-62}(d, e, f)$ Root-mean-square fluctuations (r.m.s.f.s) of $\mathrm{C}_{\alpha}$ atoms in the N-terminal regions calculated from the MD simulations.

heterotetramer in MD simulation when the N-terminal region is cleaved, then the VASH1-SVBP complex might be in the fully functional heterodimeric form. Thus, tetramer formation and N-terminal flexibility might lock VASH1 into an inactive conformation preventing substrate binding, catalysis or turnover.

There are several biochemical reports of VASH1-SVBP and VASH2-SVBP complexes. The endogenous full-length VASH1-SVBP and VASH2-SVBP complexes showed tubulin carboxypeptidase activity which was inhibited by alkyne-epoY (Aillaud et al., 2017) or genetic disruptions (Nieuwenhuis et al., 2017). The activity of recombinant full-length VASH1 and VASH2 required the presence of SVBP, and the activity was abolished by mutating the catalytic cysteines in VASH1 or VASH2 (Aillaud et al., 2017; Nieuwenhuis et al., 2017). Biochemical analysis of the truncated VASH1(52-310)-SVBP complex revealed the enzymatic properties of the VASH1cSVBP complex, with a $K_{\mathrm{m}}$ of $7.9 \mu M$ and a $k_{\text {cat }}$ of $44.5 \mathrm{~min}^{-1}$ against tubulin peptides ( $\mathrm{Li}$ et al., 2019). The difference between the full-length and truncated complexes has not yet been reported. Furthermore, there has been no report of a comparison between the activity of the heterodimer and the heterotetramer. Future biochemical characterization, including that of mutants in the N-terminal region, should reveal the role of the $\mathrm{N}$-terminus in vasohibin function.

\section{Acknowledgements}

We would like to thank Dr Kosuke Morikawa for critically reading the manuscript and Dr Kazumi Tsuda for his help in the early MD simulation analysis. The synchrotron experiments were performed under the approval of the Photon Factory Program Advisory Committee (Proposal Nos. 2016G180 and 2018G113) and on BL44XU at SPring-8 with the approval of the Japan Synchrotron Radiation Research Institute (JASRI; Proposal Nos. 2018A6838, 2018B6838 and 2019A6935).

\section{Funding information}

We acknowledge support from the collaboration project of the Platform Project for Supporting Drug Discovery and Life Science Research (Basis for Supporting Innovative Drug Discovery and Life Science Research; BINDS) from AMED (Grant No. 1739), NIG-JOINT (Grant Nos. 6A2017, 2A2018 and 85A2019) and the Cooperative Research Program of the Institute for Protein Research, Osaka University (Grant Nos. CR-17-05, CR-18-05 and CR-19-05). This work was supported by a Grant-in-Aid for Scientific Research from the Japanese Society for the Promotion of Science (Grant No. 16K07279).

\section{References}

Adamopoulos, A., Landskron, L., Heidebrecht, T., Tsakou, F., Bleijerveld, O. B., Altelaar, M., Nieuwenhuis, J., Celie, P. H. N., Brummelkamp, T. R. \& Perrakis, A. (2019). Nat. Struct. Mol. Biol. 26, 567-570.

Aillaud, C., Bosc, C., Peris, L., Bosson, A., Heemeryck, P., Van Dijk, J., Le Friec, J., Boulan, B., Vossier, F., Sanman, L. E., Syed, S., Amara, N., Couté, Y., Lafanechère, L., Denarier, E., Delphin, C., Pelletier, L., Humbert, S., Bogyo, M., Andrieux, A., Rogowski, K. \& Moutin, M.-J. (2017). Science, 358, 1448-1453. 
Berendsen, H. J. C., Postma, J. P. M., van Gunsteren, W. F., DiNola, A. \& Haak, J. R. (1984). J. Chem. Phys. 81, 3684-3690.

Case, D. A., Ben-Shalom, I. Y., Brozell, S. R., Cerutti, D. S., Cheatham, T. E. III, Cruzeiro, V. W. D., Darden, T. A., Duke, R. E., Ghoreishi, D., Gilson, M. K., Gohlke, H., Goetz, A. W., Greene, D., Harris, R., Homeyer, N., Izadi, S., Kovalenko, A., Kurtzman, T., Lee, T. S., LeGrand, S., Li, P., Lin, C., Liu, J., Luchko, T., Luo, R., Mermelstein, D. J., Merz, K. M., Miao, Y., Monard, G., Nguyen, C., Nguyen, H., Omelyan, I., Onufriev, A., Pan, F., Qi, R., Roe, D. R., Roitberg, A., Sagui, C., Schott-Verdugo, S., Shen, J., Simmerling, C. L., Smith, J., Salomon-Ferrer, R., Swails, J., Walker, R. C., Wang, J., Wei, H., Wolf, R. M., Wu, X., Xiao, L., York, D. M. \& Kollman, P. A. (2018). Amber18. University of California, San Francisco.

Dunn, B. M. (2002). Chem. Rev. 102, 4431-4458.

Emsley, P., Lohkamp, B., Scott, W. G. \& Cowtan, K. (2010). Acta Cryst. D66, 486-501.

Humphrey, W., Dalke, A. \& Schulten, K. (1996). J. Mol. Graph. 14, 33-38.

Kabsch, W. (2010). Acta Cryst. D66, 125-132.

Kadonosono, T., Yimchuen, W., Tsubaki, T., Shiozawa, T., Suzuki, Y., Kuchimaru, T., Sato, Y. \& Kizaka-Kondoh, S. (2017). Protein Sci. 26, 452-463.

Li, F., Hu, Y., Qi, S., Luo, X. \& Yu, H. (2019). Nat. Struct. Mol. Biol. 26, 583-591.

Liao, S., Rajendraprasad, G., Wang, N., Eibes, S., Gao, J., Yu, H., Wu, G., Tu, X., Huang, H., Barisic, M. \& Xu, C. (2019). Cell Res. 29, 533-547.

Liebschner, D., Afonine, P. V., Baker, M. L., Bunkóczi, G., Chen, V. B., Croll, T. I., Hintze, B., Hung, L.-W., Jain, S., McCoy, A. J., Moriarty, N. W., Oeffner, R. D., Poon, B. K., Prisant, M. G., Read, R. J., Richardson, J. S., Richardson, D. C., Sammito, M. D., Sobolev, O. V., Stockwell, D. H., Terwilliger, T. C., Urzhumtsev, A. G., Videau, L. L., Williams, C. J. \& Adams, P. D. (2019). Acta Cryst. D75, 861-877.

Liu, X., Wang, H., Zhu, J., Xie, Y., Liang, X., Chen, Z., Feng, Y. \& Zhang, Y. (2019). Cell. Discov. 5, 65.
Loncharich, R. J., Brooks, B. R. \& Pastor, R. W. (1992). Biopolymers, 32, 523-535.

Maier, J. A., Martinez, C., Kasavajhala, K., Wickstrom, L., Hauser, K. E. \& Simmerling, C. (2015). J. Chem. Theory Comput. 11, 36963713.

Morales, R., Watier, Y. \& Böcskei, Z. (2012). J. Mol. Biol. 421, 100111.

Nieuwenhuis, J., Adamopoulos, A., Bleijerveld, O. B., Mazouzi, A., Stickel, E., Celie, P., Altelaar, M., Knipscheer, P., Perrakis, A., Blomen, V. A. \& Brummelkamp, T. R. (2017). Science, 358, $1453-$ 1456.

Pagnamenta, A. T., Heemeryck, P., Martin, H. C., Bosc, C., Peris, L., Uszynski, I., Gory-Fauré, S., Couly, S., Deshpande, C., Siddiqui, A., Elmonairy, A. A., WGS500 Consortium, Genomics England Research Consortium, Jayawant, S., Murthy, S., Walker, I., Loong, L., Bauer, P., Vossier, F., Denarier, E., Maurice, T., Barbier, E. L., Deloulme, J.-C., Taylor, J. C., Blair, E. M., Andrieux, A. \& Moutin, M.-J. (2019). Hum. Mol. Genet. 28, 3391-3405.

Ryckaert, J.-P., Ciccotti, G. \& Berendsen, H. J. C. (1977). J. Comput. Phys. 23, 327-341.

Sanchez-Pulido, L. \& Ponting, C. P. (2016). Bioinformatics, 32, 14411445.

Sato, Y. (2013). J. Biochem. 153, 5-11.

Sonoda, H., Ohta, H., Watanabe, K., Yamashita, H., Kimura, H. \& Sato, Y. (2006). Biochem. Biophys. Res. Commun. 342, 640646.

Suzuki, Y., Kobayashi, M., Miyashita, H., Ohta, H., Sonoda, H. \& Sato, Y. (2010). J. Cell Sci. 123, 3094-3101.

Tanabe, K., Sato, Y. \& Wada, J. (2018). Int. J. Mol. Sci. 19, 1859.

Wang, N., Bosc, C., Choi, S. R., Boulan, B., Peris, L., Olieric, N., Bao, H., Krichen, F., Chen, L., Andrieux, A., Olieric, V., Moutin, M.-J., Steinmetz, M. O. \& Huang, H. (2019). Nat. Struct. Mol. Biol. 26, 571-582.

Zhou, C., Yan, L., Zhang, W. \& Liu, Z. (2019). Nat. Commun. 10, 3212. 